\title{
The diagnosis and treatment of dermatitis herpetiformis
}

This article was published in the following Dove Press journal:

Clinical, Cosmetic and Investigational Dermatology

13 May 2015

Number of times this article has been viewed

\author{
Emiliano Antiga \\ Marzia Caproni \\ Department of Surgery and \\ Translational Medicine, Section of \\ Dermatology, University of Florence, \\ Florence, Italy
}

\begin{abstract}
Dermatitis herpetiformis (DH) is an inflammatory cutaneous disease with a chronic relapsing course, pruritic polymorphic lesions, and typical histopathological and immunopathological findings. According to several evidences, DH is considered the specific cutaneous manifestation of celiac disease, and the most recent guidelines of celiac disease have stated that, in celiac patients with a proven $\mathrm{DH}$, a duodenal biopsy is unnecessary for the diagnosis. In this review, the most recent data about the diagnosis and the management of $\mathrm{DH}$ have been reported and discussed. In particular, in patients with clinical and/or histopathological findings suggestive for DH, the finding of granular IgA deposits along the dermal-epidermal junction or at the papillary tips by direct immunofluorescence (DIF) assay, together with positive results for anti-tissue transglutaminase antibody testing, allows the diagnosis. Thereafter, a gluten-free diet should be started in association with drugs, such as dapsone, that are able to control the skin manifestations during the first phases of the diet. In conclusion, although DH is a rare autoimmune disease with specific immunopathological alterations at the skin level, its importance goes beyond the skin itself and may have a big impact on the general health status and the quality of life of the patients.
\end{abstract}

Keywords: dermatitis herpetiformis, celiac disease, diagnosis, treatment, autoimmune disease, inflammatory cutaneous disease

\section{Introduction}

Dermatitis herpetiformis (DH) is an inflammatory cutaneous disease with a chronic relapsing course, pruritic polymorphic lesions, and typical histopathological and immunopathological findings.

According to several evidences, DH is considered the specific cutaneous manifestation of celiac disease (CD). In fact, both diseases occur in gluten-sensitive individuals, share the same HLA haplotypes (DQ2 and DQ8), and improve following the administration of a gluten-free diet. ${ }^{1}$ Moreover, patients with DH show typical CD alterations at the small bowel biopsy (ranging from villous atrophy to augmented presence of intraepithelial lymphocytes [IELs]) almost in all the cases, as well as the generation of circulating autoantibodies to tissue transglutaminase (tTG).

$\mathrm{DH}$ is predominately a disorder of Caucasians, ${ }^{2}$ although Japanese cases are increasingly reported. ${ }^{3}$ The incidence of the disease was found to be 11.5 per 100,000 in Scotland ${ }^{4}$ and ranging from 19.6 to 39.2 per 100,000 in Sweden. ${ }^{5}$ In a recent study from Finland, the prevalence of DH was found to be 75.3 per 100,000 (eight times lower than the prevalence of CD in that area), while the annual incidence was found to 3.5 per 100,000 over the period $1980-2009$, showing a decrease in the last years. ${ }^{6}$
Department of Surgery and Translational Medicine, Section of Dermatology, University of Florence, Viale Michelangelo 4I, 50125 Florence, Italy

Tel +39055 6939664

Fax +39055 6939598

Email emiliano.antiga@unifi.it 
DH usually presents in the fourth and fifth decades, although individuals of any age can be affected. In a recent study from our group investigating 159 patients with $\mathrm{DH}$, approximately $27 \%$ of the patients were below the age of 10 , and $36 \%$ below the age of 20 , showing that, at least in Italy, pediatric DH is more common than expected in other countries. ${ }^{7}$

In 2009, the guidelines for the management of patients with DH were published by our group. ${ }^{1}$ However, according to recent literature, several new findings have been reported about the clinical and immunopathological features of $\mathrm{DH}$; moreover, the novel guidelines for the management of CD from the European Society for Pediatric Gastroenterology, Hepatology, and Nutrition (ESPGHAN) were developed in 2012. ${ }^{8}$ Therefore, an update on the diagnosis and treatment of DH would be helpful to improve the care of the patients.

Accordingly, in the next paragraphs, the clinical and the immunopathological features that can help in the diagnosis of $\mathrm{DH}$ are reported. Moreover, the management of the disease, which is based both on a gluten-free diet and on medications that can help control DH in the inflammatory phases, as well as its follow-up are discussed.

\section{Clinical features}

DH usually presents with symmetrical, grouped polymorphic lesions consisting of erythema, urticarial plaques, and papules, ${ }^{2,9-11}$ involving the extensor surfaces of the knees, elbows, shoulders, buttocks, sacral region, neck, face, and scalp. By contrast, herpetiform vesicles, which reflect the name of the disease, may occur later or are often immediately excoriated, resulting in erosions, crusted papules, or areas of postinflammatory dyschromia, and are usually not seen in the patients. Itching of variable intensity and scratching and burning sensation immediately preceding the development of lesions are common. ${ }^{2,9-11}$

Together with these manifestations, several atypical presentations have been reported in patients with $\mathrm{DH}$, including purpuric lesions resembling petechiae on hands and feet, ${ }^{12-20}$ leukocytoclastic vasculitis-like appearance, ${ }^{21}$ palmo-plantar keratosis, ${ }^{22}$ wheals of chronic urticaria, ${ }^{23}$ and lesions mimicking prurigo pigmentosa. ${ }^{24}$ Interestingly, in some cases patients may show erythema or severe pruritus alone, making the diagnosis challenging. ${ }^{25}$ Finally, patients with DH may present the clinical manifestations associated with gastrointestinal malabsorption, although less frequently than in CD.

Clinically, the main differential diagnoses in children are atopic dermatitis, scabies, papular urticaria, and impetigo, whereas eczema, other autoimmune blistering diseases (especially $\operatorname{IgA}$ linear disease and bullous pemphigoid), nodular prurigo, urticaria, and polymorphic erythema should be considered in adults. ${ }^{1}$

\section{Histopathological findings}

The typical histopathological findings in the lesional skin of patients with DH consist of subepidermal vesicles and blisters associated with accumulation of neutrophils at the papillary tips. ${ }^{2,10,11}$ Sometimes, eosinophils can be found within the inflammatory infiltrate, ${ }^{26}$ making difficult the differential diagnosis with bullous pemphigoid.

The histopathology of a DH skin lesion can be evocative, but it is not diagnostic, since other bullous diseases, including linear IgA dermatosis, epidermolysis bullosa acquisita, and others may show similar findings. ${ }^{1,2,10}$ Moreover, as demonstrated by Warren and Cockerell, ${ }^{27}$ the histopathologic picture is unspecific in approximately $35 \%-40 \%$ of the cases, revealing only perivascular lymphocytic infiltrate and minimal inflammation in dermal papillae. Thus, to achieve the diagnosis, histopathologic examination should be always performed in combination with DIF of perilesional skin, which represent the gold standard for the diagnosis of $\mathrm{DH} .{ }^{1,2}$

\section{Direct immunofluorescence}

As just stated, DIF of uninvolved skin collected in the perilesional site is the gold standard for the diagnosis of DH. ${ }^{1,2}$ Two specific patterns of DIF are possible: 1) granular deposits at the dermal papillae and 2) granular deposits along the basement membrane. Sometimes, a combination of both patterns, consisting in granular IgA deposition along the basement membrane with accentuation at the papillary tips, may be present. ${ }^{1,2}$ Recently, a third pattern consisting of fibrillar IgA deposits mainly located at the papillary tips has been described. ${ }^{28}$ Such a pattern is often seen in Japanese patients with $\mathrm{DH}$, where it is described in up to $50 \%$ of the cases. ${ }^{3}$

Other kinds of immune deposits that can be found by DIF are the presence of perivascular IgA deposits in the upper dermis, as well as of granular IgM or C3 deposits at the dermal-epidermal junction and/or at the dermal papillae.

DIF has a sensitivity and a specificity close to $100 \%$ for the diagnosis of DH. Moreover, according to the ESPGHAN guidelines for $\mathrm{CD}$, a positive DIF in a patient with suspected $\mathrm{DH}$ allows for the diagnosis of CD without the need of duodenal biopsy. ${ }^{8}$ DIF should be performed on uninvolved perilesional skin, since in skin lesions IgA can be removed by inflammatory cells. Moreover, patients must be on normal diet, because IgA deposits can disappear from the skin in period of times variable from weeks to months in patients 
on a gluten-free diet. If the patient is on a gluten-free diet, a normal gluten-containing diet should be administered and the biopsy taken after at least 1 month.

In the case of negative results for DIF in patients with a high clinical suspicion of DH, the site of the biopsy should be reconsidered and another specimen should be taken from uninvolved perilesional skin. Very rarely, cases of patients with DH showing negative DIF results are reported in the literature. ${ }^{29-31}$ In such cases, the combination of clinical, histopathological, and serological data, together with all the examination needed for $\mathrm{CD}$, can help make the diagnosis.

\section{Serologic analysis}

Patients with DH usually show the specific antibodies that can be found in patients with CD. Among them, IgA anti-tTG antibodies are considered the most sensitive and specific ones and should be tested as the first-line serologic investigation in patients with a suspected DH. Some patients may have IgA deficiency; so the total serum IgA should be tested to exclude false-negative results from the serological investigation.

IgA anti-endomysium antibodies (EMAs), IgA and IgG anti-deamidated synthetic gliadin-derived peptides (DGP), and IgA anti-epidermal transglutaminase (eTG) antibodies are considered specific and sensitive serologic markers for DH. Finally, other kinds of antibodies are currently under investigation in both patients with $\mathrm{DH}$ and $\mathrm{CD}$. The main features of the antibodies that can be detected in patients with $\mathrm{DH}$ are reported in what follows.

\section{Anti-tTG antibodies}

Anti-tTG antibodies belong to the IgA1 subclass and represent a good marker of intestinal damage and of gluten-free diet adherence in patients with the DH/CD spectrum. ${ }^{32}$ The commercially available ELISA (enzyme-linked immunosorbent assay) kits have a sensitivity ranging from $47 \%$ to $95 \%$ and a specificity higher than $90 \%$ for the diagnosis of $\mathrm{DH}^{33-36}$

Since they are detected with a validated immunoenzymatic assay that is quite cheap and easy to perform, they are currently considered the most useful serological marker in celiac patients.

\section{EMA}

Even EMA belong to the IgA1 subclass, and are directed against primate smooth muscle reticular connective tissue. The detection of EMA is based on an indirect immunofluorescence assay on monkey esophagus. EMA testing has shown a specificity close to $100 \%$ and a sensitivity ranging from $52 \%$ to $100 \%$ for the diagnosis of $\mathrm{DH} .{ }^{33-37}$ As for antitTG, EMA are usually absent in patients on a gluten-free diet and thus represent a useful diet-compliance marker in celiac patients. ${ }^{9-11,38}$ However, since it is more expensive, time-consuming, and operator-dependent than the anti-tTG ELISA,${ }^{38}$ EMA testing should be performed only in doubtful cases.

\section{Anti-DGP antibodies}

In patients with $C D$, anti-DGP antibodies show lower sensitivity and specificity than anti-tTG and EMA..$^{39,40}$ Their role as a useful marker of $\mathrm{CD}$ in patients below the age of 2 , in whom the other antibodies are often absent, is still under debate. ${ }^{41-43}$ Few reports are present in the literature about anti-DGP antibodies in patients with $\mathrm{DH}$, showing results similar to those with anti-tTG ones. ${ }^{4446}$ Therefore, in clinical practice, anti-DGP antibodies should be tested only in doubtful cases.

\section{Anti-eTG antibodies}

Recent evidence has demonstrated that patients with $\mathrm{DH}$ have antibodies directed against eTG, which is considered the specific antigen of $\mathrm{DH} .{ }^{47}$ Anti-eTG antibodies show for $\mathrm{DH}$ a sensitivity ranging from $52 \%$ to $100 \%$, and a specificity higher than $90 \%,{ }^{46,48-50}$ thus giving results similar to those with anti-tTG antibodies.

Since the ELISA kit to detect anti-eTG antibodies is not widely available in all the laboratories, to date they are tested only for research purposes and not for the clinical management of the patients.

\section{Other antibodies}

Other antibodies that are currently under investigation as markers for $\mathrm{CD}$ and/or DH are the anti-neoepitope tTG antibodies ${ }^{46}$ and the anti-GAF3X antibodies. ${ }^{51}$ Although they might be good markers for $\mathrm{DH}$, further studies are required to confirm their usefulness as tools for the diagnosis of the disease.

\section{HLA haplotypes testing}

As in CD, virtually all patients with DH carry either HLADQ2 (DQA1*05, DQB1*02) or HLADQ8 (DQB1*0302). ${ }^{1}$

Thus, the presence of these alleles provides a sensitivity of close to $100 \%$ for $\mathrm{DE}$ and a very high negative predictive value for the disease (ie, if individuals lack the relevant disease-associated alleles, CD can be excluded). By contrast, since $30 \%-40 \%$ of the general population carry such HLA alleles, the specificity of such a test is very low. ${ }^{32}$ 
Therefore, HLA testing, if negative, may be helpful in excluding the diagnosis of DH. It can also be helpful as a screening tool for patients with high risk for $\mathrm{CD}$, including first-degree relatives of patients with $\mathrm{CD}$.

\section{Small bowel biopsy}

As in CD, patients with DH show intestinal involvement that can be documented by histopathology in most cases. The features include partial-to-total villous atrophy, elongated crypts, decreased villus/crypt ratio, increased mitotic index in the crypts, increased IELs density, increased IEL mitotic index, infiltration of plasma cells, lymphocytes, mast cells, and eosinophils and basophils into the lamina propria. ${ }^{8}$ However, in general, the histopathological alterations found in patients with $\mathrm{DH}$ are milder than those found in patients with CD.

According to the Marsh classification modified by Oberhuber et $\mathrm{al}^{52}$ the intestinal damage in $\mathrm{CD}$ patients can be divided into different stages, ranging from the normal mucosa to villous atrophy (Marsh III).

Since DH can be considered as CD of the skin, in a patient with a proven diagnosis of $\mathrm{DH}$, duodenal biopsy is no longer required to confirm the diagnosis, as stated in recent guidelines. ${ }^{8}$ However, in doubtful DH cases (eg, with atypical clinical or immunopathological features), all the measures that are necessary to make a diagnosis of CD, including duodenal biopsy, should be performed. Moreover, duodenal biopsy should be performed in case of suspected gastrointestinal complications, including lymphoma.

\section{Diagnostic algorithm}

In patients with clinical and/or histopathological findings suggestive for DH, a biopsy of perilesional skin for DIF should be performed and serum samples should be collected to test anti-tTG antibodies (together with total IgA dosing). Then, basing on the evidences reported earlier, the diagnostic algorithm should be as follows (Figure 1):

1) In case of typical findings from DIF (ie, granular IgA deposits at the dermal-epidermal junction or at the papillary tips) and of positive anti-tTG testing, the diagnosis of $\mathrm{DH}$ and, accordingly, of CD can be confirmed. Therefore, treatment and monitoring of DH should be managed (see text that follows).

2) In case of typical DIF results, but with negative anti-tTG antibodies, HLA DQ2/DQ8 testing is suggested. If negative, DH can be excluded, but if positive, patients should be further investigated. In particular, EMA and anti-DGP antibodies should be tested in order to exclude a previous false-negative result for anti-tTG antibodies. If EMA or anti-DGP antibodies are positive, DH can be confirmed.

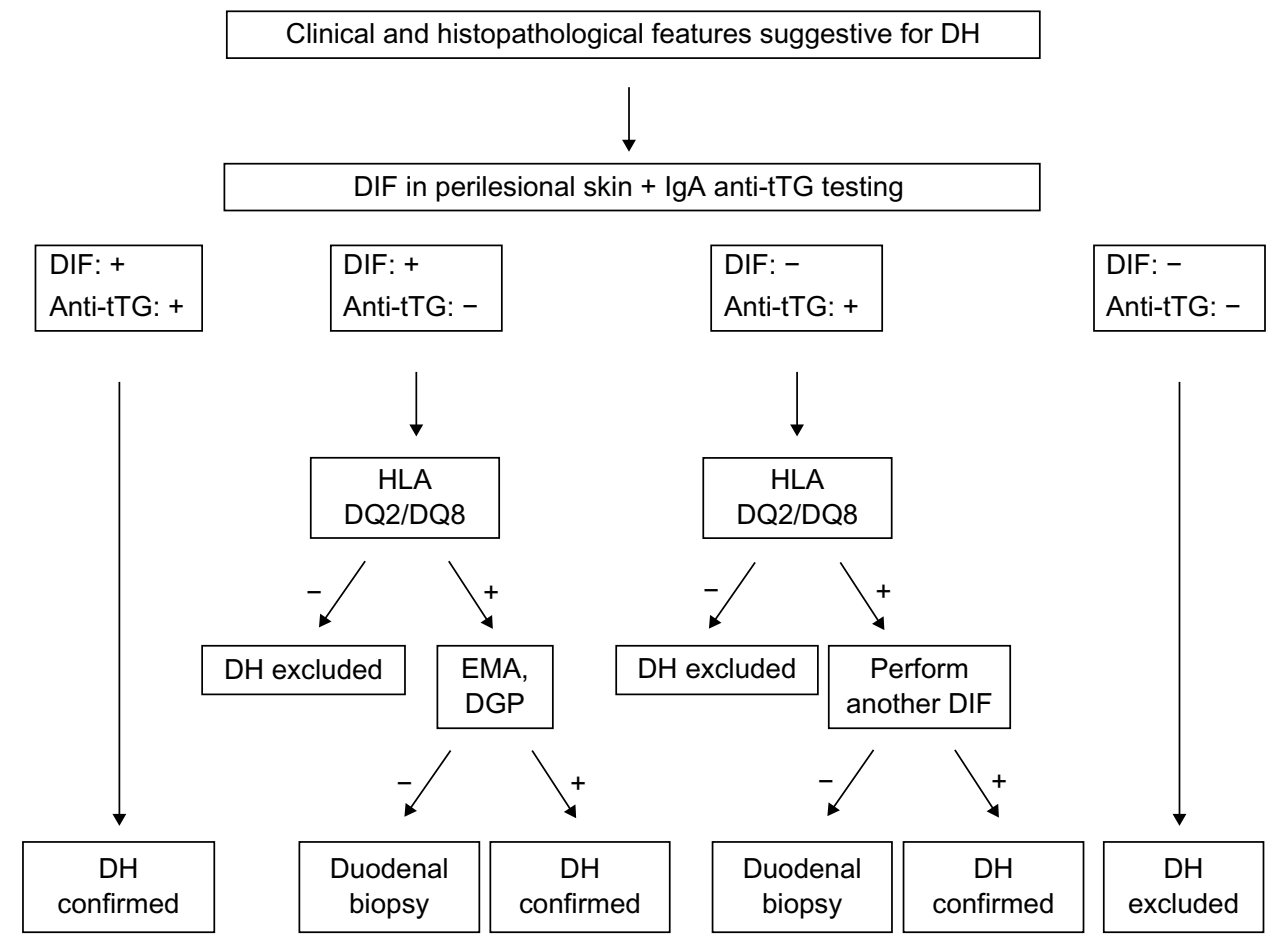

Figure I Diagnostic algorithm for patients with dermatitis herpetiformis.

Abbreviations: Anti-tTG, anti-tissue transglutaminase antibodies; DGP, anti-deamidated gliadin peptide antibodies; DH, dermatitis herpetiformis; DIF, direct immunofluorescence; EMA, anti-endomysium antibodies; HLA, human leukocyte antigen. 
If negative, the guidelines for the diagnosis of CD should be followed, ${ }^{8,53,54}$ including the implementation of duodenal biopsy, in order to confirm the intestinal involvement prior to starting a gluten-free diet.

3) In case of negative DIF and the presence of anti-tTG antibodies, HLA DQ2/DQ8 testing is suggested. If negative, $\mathrm{DH}$ can be excluded, but if positive, patients should be further investigated. First of all, a new skin biopsy of perilesional skin for DIF should be performed, in order to exclude false-negative results due to wrong sample collection in the previous skin biopsy. If the new DIF shows typical DH findings, the diagnosis can be confirmed. If DIF result is again negative, according to the guidelines for the diagnosis of $\mathrm{CD}$, a duodenal biopsy is suggested. ${ }^{8,53,54}$

4) In case of negative results both for DIF and for anti-tTG testing, DH can be excluded and the clinical and histopathological findings of the patients should be revised in order to achieve a different diagnosis.

\section{Treatment}

As previously stated, DH is considered the specific cutaneous manifestation of CD; therefore, a lifelong gluten-free diet is the first-choice treatment of the disease. However, in the first month after the diagnosis or in the inflammatory phases of the disease, in which a gluten-free diet alone would not be enough to control the symptoms, several drugs can be used for variable periods of time, including dapsone, sulfones or steroids.

\section{Gluten-free diet}

A strict gluten-free diet is the mainstay for treatment of the spectrum DH/CD. The level of gluten allowed is $<20$ ppm (gluten-free); however, in some countries, products with $<100$ ppm (very low gluten) are allowed.

Gluten-free diet is able to resolve both the gastrointestinal and the cutaneous manifestations, as well as to prevent the development of lymphomas and other diseases associated with gluten-induced enteropathy and malabsorption.

Gluten-free diet alleviates gastrointestinal symptoms in an average of 3-6 months, which is much more rapidly than what happens with the rash; in fact, it takes an average of 1-2 years of a gluten-free diet for the complete resolution of the cutaneous lesions, which invariably recur within 12 weeks after the reintroduction of gluten. IgA antibodies may disappear from the dermal-epidermal junction after many years of a strict gluten-free diet. ${ }^{55-59}$

Gluten is present in cereal species of the tribe Triticeae, which includes wheat, rye, and barley. ${ }^{56}$ Although in the past the basis of a gluten-free diet was the avoidance of all gluten-containing cereals, including wheat, barley, rye, and oats (mnemonic BROW), recently, some authors have demonstrated that oats belonging to the Avenae tribe can be safely consumed by celiac patients. ${ }^{60-62}$ However, only oats known to be pure and not contaminated in any way with wheat, barley, or rye (which is the case of the majority of commercially available oats) can be safely consumed. ${ }^{2}$

As reviewed by Hischenhuber et al, ${ }^{63}$ evidence-based studies show that a diet including industrially purified glutenfree wheat starch-based flours is safe for patients with DH/ CD spectrum and the small-intestinal mucosa heals and stays long-term morphologically normal. ${ }^{62}$

After following $133 \mathrm{DH}$ patients, Garioch et $\mathrm{al}^{57}$ reported several advantages of a gluten-free diet, including a reduced need for medication to treat the cutaneous manifestations, the resolution of enteropathy, a general feeling of well-being, and a protective effect against development of lymphoma. Moreover, although further evidences are required, a glutenfree diet might be helpful even in the prevention of the occurrence of DH/CD-related autoimmune disorders.

Recently, a few studies have suggested that DH can go into remission in up to $20 \%$ of the cases, ${ }^{64,65}$ and therefore, clinicians should continually reevaluate the need for a gluten-free diet for their patients with well-controlled DH. ${ }^{65}$ However, since a gluten-free diet in patients with $\mathrm{DH}$ should not be considered a mere symptomatic approach to treat skin manifestation, but also the way to control and to prevent all the complications of $\mathrm{CD}$, other studies are required to confirm whether the gluten-free diet can be safely discontinued. ${ }^{66}$ Accordingly, lifelong commitment to a gluten-free diet is considered essential by gastroenterologists in $\mathrm{CD}$ and offers the patient a much better quality of life, avoidance of most complications, and an effective cure. ${ }^{67}$

Even though a gluten-free diet offers many benefits in the management of $\mathrm{DH}$, in practice, it is not well adopted by many DH patients. In fact, it requires scrupulous monitoring of all ingested foods, it is time-consuming, and socially restricting. ${ }^{56}$ Gluten-free products are not widely available and are more expensive than their gluten-containing counterparts; moreover, contamination with small amounts of gluten is possible. ${ }^{68}$ It has become evident that $20 \%-80 \%$ of patients with CD may continue to suffer from symptoms and still have a gluten-induced manifest mucosal lesion of Marsh II and III classes, and accordingly, some patients with DH still have skin manifestations, despite adherence to a gluten-free diet. ${ }^{62,69}$ Therefore, treatments alternative or integrative to the gluten-free diet in order to minimize cross-contamination 
accidents typically occurring outside patients' households would represent desirable interventions to minimize the risk of complications associated with prolonged gluten exposure in subjects affected by $\mathrm{CD}$ and $\mathrm{DH}{ }^{68}$

\section{Dapsone}

Although no reports from randomized controlled trials are present in the literature about its use, dapsone is considered a valid therapeutic option for patients with DH during the 6to 24-month period until the gluten-free diet is effective. ${ }^{70-77}$ The starting dose should be $50 \mathrm{mg} / \mathrm{d}$ in order to minimize the potential side effects. Then the dosage can be increased up to $200 \mathrm{mg} / \mathrm{d}$ until the disease is under control; in the maintenance phase, $0.5-1 \mathrm{mg} / \mathrm{kg} / \mathrm{d}$ generally can control itching and the development of new skin lesions..$^{71-78}$

As just reported, several side effects are associated with dapsone use. They are usually dose-dependent and more frequent in patients with comorbidities, such as anemia, cardiopulmonary disease, glucose-6-phosphate dehydrogenase deficiency. ${ }^{1,73}$

They are classified into toxic, including hemolytic anemia (that usually occurs within the first 2 weeks) and methemoglobinemia, and idiosyncratic. Among the latter, dapsone hypersensitivity syndrome is considered the most severe and occurs within 2-6 weeks in approximately $5 \%$ of the patients, consisting of fever, photosensitivity, rash, malaise, lymphadenopathy, neurological effects, nephropathy, hypothyroidism, gastrointestinal symptoms and liver involvement up to hepatic failure in some cases. ${ }^{75}$

Owing to these side effects, patients using dapsone should be carefully monitored. Before starting the therapy, complete blood count, glucose-6-phosphate dehydrogenase, methemoglobinemia, liver and renal functions, as well as urinalysis should be investigated. Then, patients should be reevaluated every week for the first month to monitor anemia, methemoglobinemia, and neuropathy symptoms. After the first months, complete blood count should be performed twice a month for the following 2 months and then every 3 months (together with liver and renal function testing). ${ }^{78}$

\section{Sulfasalazine, sulfapyridine, and sulfamethoxypyridazine}

If dapsone fails to control the symptoms or in case of adverse effects, sulfasalazine ( $1-2 \mathrm{~g} / \mathrm{d})$, sulfapyridine ( $2-4 \mathrm{~g} / \mathrm{d})$, and sulfamethoxypyridazine $(0.25-1.5 \mathrm{~g} / \mathrm{d})$ can be valid alternatives for the treatment of patients with $\mathrm{DH},{ }^{2,79,80}$

All the three drugs share similar adverse effects, consisting of gastrointestinal upset (with nausea, anorexia, and vomiting), hypersensitivity drug reactions, hemolytic anemia, proteinuria, and crystalluria. Therefore, before starting the treatment, full blood count with differential and urine microscopy with urinalysis should be carried out. The same examination should be repeated monthly after the first 3 months and thereafter every 6 months.

The enteric-coated forms of the drugs, which are currently available, can prevent the symptoms associated with the gastrointestinal upset. ${ }^{79,80}$

\section{Other drugs}

Other drugs can be used to control the skin symptoms in patients with DH. Among them, potent (betamethasone valerate or dipropionate) or very potent (clobetasol propionate) topical steroids are helpful in cases with localized disease to reduce pruritus and the appearance of new lesions. ${ }^{78}$ Accordingly, systemic steroids or antihistamines can control, at least in part, itching and burning sensation, although their effectiveness is considered quite low. ${ }^{78}$

Other drugs that have been shown to be effective in some reports are topical dapsone, immunosuppressors such as cyclosporin A or azathioprine, colchicine, heparin, tetracyclines, nicotinamide, mycophenolate, and rituximab. ${ }^{81-88}$

Finally, several new experimental approaches for the treatment of CD are currently under investigation, including the use of engineered grains and inhibitory gliadin peptides, immunomodulatory strategies to prevent the development of an immune response against gluten, the correction of the intestinal barrier defect, and others (reviewed in Fasano et $\mathrm{a}^{68}$ ). As happens with a gluten-free diet, such approaches might be helpful even in the control of DH skin manifestations.

\section{Follow-up}

Since DH is associated with CD, patients should be monitored following the recent guidelines for such a disease. ${ }^{8,53,54}$ Patients with DH should be evaluated at regular intervals (6 months after diagnosis and then yearly) by a multidisciplinary team involving at least a physician and a dietitian. The purposes of these visits are to assess the compliance with the gluten-free diet and the presence of dyslipidemia, and to evaluate the possible development of intestinal malabsorption and/or celiac-related conditions, including other autoimmune diseases and complications such as refractory $\mathrm{CD}$, ulcerative ileitis, celiac sprue, or lymphoma. Among the autoimmune or immune-mediated associated diseases, Hashimoto thyroiditis, insulin-dependent diabetes mellitus, pernicious anemia, multiple sclerosis, Sjögren syndrome, lupus erythematous, 
rheumatoid arthritis, vitiligo, and psoriasis are the most frequently reported, and should be investigated in patients with familiar history or evocative clinical signs. ${ }^{87}$

Together with the visits, laboratory investigations, including immunological assessment, celiac-specific antibodies, and evaluation of intestinal malabsorption, should be performed. It should be remarked that there are no clear guidelines as to the optimal means to monitor adherence to a gluten-free diet. In fact, serological investigations (ie, antitTG or EMA) are considered to be sensitive for major, but not for minor, transient dietary indiscretions. ${ }^{40}$

\section{Conclusion}

In this review, the most recent data about the diagnosis and the management of $\mathrm{DH}$ have been reported and discussed. Although DH is a rare autoimmune disease with specific immunopathological alterations at the skin level, ${ }^{89}$ its importance goes beyond the skin itself. In fact, $\mathrm{DH}$ is considered a specific manifestation of gluten-sensitive enteropathy, and the National Institute of Health $^{90}$ as well as the most recent ESPGHAN guidelines ${ }^{8}$ stated that a duodenal biopsy is unnecessary for the diagnosis in celiac patients with a proven $\mathrm{DH}$. Therefore, not to miss a diagnosis of $\mathrm{DH}$ would allow the prompt introduction of a gluten-free diet, to prevent all the complications that are associated with $\mathrm{CD}$ and to improve the general health status as well as the quality of life of the patients.

\section{Disclosure}

The authors report no conflicts of interest in this work.

\section{References}

1. Caproni M, Antiga E, Melani L, Fabbri P; The Italian Group for Cutaneous Immunopathology. Guidelines for the diagnosis and treatment of dermatitis herpetiformis. J Eur Acad Dermatol Venereol. 2009;23(6):633-638.

2. Fry L. Dermatitis herpetiformis: problems, progress and prospects. Eur J Dermatol. 2002;12(6):523-531.

3. Ohata C, Ishii N, Hamada T, et al. Distinct characteristics in Japanese dermatitis herpetiformis: a review of all 91 Japanese patients over the last 35 years. Clin Dev Immunol. 2012;2012:562168.

4. Gawkrodger DJ, Blackwell JN, Gilmour HM, et al. Dermatitis herpetiformis diagnosis diet and demography. Gut. 1984;25(2):151-157.

5. Mobacken H, Kastrup W, Nilsson LA. Incidence and prevalence of dermatitis herpetiformis in Sweden. Arch Derm Venereol. 1984;64(5): 400-404.

6. Salmi TT, Hervonen K, Kautiainen H, Collin P, Reunala T. Prevalence and incidence of dermatitis herpetiformis: a 40-year prospective study from Finland. Br J Dermatol. 2011;165(2):354-359.

7. Antiga E, Verdelli A, Calabrò A, Fabbri P, Caproni M. Clinical and immunopathological features of 159 patients with dermatitis herpetiformis: an Italian experience. G Ital Dermatol Venereol. 2013;148(2):163-169.

8. Husby S, Koletzko S, Korponay-Szabó IR, et al. European Society for Pediatric Gastroenterology, Hepatology, and Nutrition guidelines for the diagnosis of coeliac disease. J Pediatr Gastroenterol Nutr. 2012;54(1): $136-160$.
9. Yeh SW, Ahmed B, Sami N, Ahmed AR. Blistering disorders: diagnosis and treatment. Dermatol Ther. 2003;16(3):214-223.

10. Nicolas ME, Krause PK, Gibson LE, Murray JA. Dermatitis herpetiformis. Int J Dermatol. 2003;42(8):588-600.

11. Fabbri P, Caproni M. Dermatitis herpetiformis. Orphanet Encyclopedia, February 2005:1-4. Available from: https://www.orpha.net/data/patho/ GB/uk-DermatitisHerpetiformis.pdf. Accessed December 12, 2014.

12. Marks R, Jones EW. Purpura in dermatitis herpetiformis. Br J Dermatol. 1971;84(4):386-388.

13. Moulin G, Barrut D, Franc MP Viornery P, Knezynski S. Pseudopurpuric palmar localizations of herpetiform dermatitis. Ann Dermatol Venereol. 1983;110:121-126.

14. Karpati S, Torok E, Kosnai I. Discrete palmar and plantar symptoms in children with dermatitis herpetiformis Duhring. Cutis. 1986;37(3): 184-187.

15. Pierce DK, Purcell SM, Spielvogel RL. Purpuric papules and vesicles of the palms in dermatitis herpetiformis. J Am Acad Dermatol. 1987;16(6):1274-1276.

16. Rutten A, Goos M. Palmoplantar purpura in Duhring's herpetiform dermatitis. Hautarzt. 1989;40(10):640-643.

17. Hofmann SC, Nashan D, Bruckner-Tuderman L. Petechiae on the fingertips as presenting symptom of dermatitis herpetiformis Duhring. J Eur Acad Dermatol Venereol. 2009;23(6):732-733.

18. Flann S, Degiovanni C, Derrick EK, Munn SE. Two cases of palmar petechiae as a presentation of dermatitis herpetiformis. Clin Exp Dermatol. 2010;35(2):206-208.

19. Heinlin J, Knoppke B, Kohl E, Landthaler M, Karrer S. Dermatitis herpetiformis presenting as digital petechiae. Pediatr Dermatol. 2012;29(2):209-212.

20. Bonciolini V, Bonciani D, Verdelli A, et al. Newly described clinical and immunopathological feature of dermatitis herpetiformis. Clin Dev Immunol. 2012;2012:967974.

21. Naylor E, Atwater A, Selim MA, Hall R, Puri PK. Leukocytoclastic vasculitis as the presenting feature of dermatitis herpetiformis. Arch Dermatol. 2011;147(11):1313-1316.

22. Ohshima Y, Tamada Y, Matsumoto Y, Hashimoto T. Dermatitis herpetiformis Duhring with palmoplantar keratosis. $\mathrm{Br} J$ Dermatol. 2003;149(6):1300-1302.

23. Powell GR, Bruckner AL, Weston WL. Dermatitis herpetiformis presenting as chronic urticaria. Pediatr Dermatol. 2004;21(5):564-567.

24. Saito M, Böer A, Ishiko A, Nishikawa T. Atypical dermatitis herpetiformis: a Japanese case that presented with initial lesions mimicking prurigo pigmentosa. Clin Exp Dermatol. 2006;31(2):290-291.

25. Junkins-Hopkins JM. Dermatitis herpetiformis: pearls and pitfalls in diagnosis and management. J Am Acad Dermatol. 2010;63(3): 526-528.

26. Caproni M, Feliciani C, Fuligni A, et al. Th2-like cytokine activity in dermatitis herpetiformis. Br J Dermatol. 1998;138(2):242-247.

27. Warren SJ, Cockerell CJ. Characterization of a subgroup of patients with dermatitis herpetiformis with nonclassical histologic features. $\mathrm{Am}$ $J$ Dermatopathol. 2002;24(4):305-308.

28. Ko CJ, Colegio OR, Moss JE, McNiff JM. Fibrillar IgA deposition in dermatitis herpetiformis - an underreported pattern with potential clinical significance. J Cutan Pathol. 2010;37(4):475-477.

29. Beutner EH, Baughman RD, Austin BM, Plunkett RW, Binder WL. A case of dermatitis herpetiformis with IgA endomysial antibodies but negative direct immunofluorescent findings. J Am Acad Dermatol. 2000;43(2 Pt 2):329-332.

30. Sousa L, Bajanca R, Cabral J, Fiadeiro T. Dermatitis herpetiformis: should direct immunofluorescence be the only diagnostic criterion? Pediatr Dermatol. 2002;19(4):336-339.

31. Huber C, Trüeb RM, French LE, Hafner J. Negative direct immunofluorescence and nonspecific histology do not exclude the diagnosis of dermatitis herpetiformis Duhring. Int J Dermatol. 2013;52(2):248-249.

32. Rostom A, Murray JA, Kagnoff MF. American Gastroenterological Association (AGA) Institute technical review on the diagnosis and management of celiac disease. Gastroenterology. 2006;131(6):1981-2002. 
33. Porter WM, Unsworth DJ, Lock RJ, Hardman CM, Baker BS, Fry L. Tissue transglutaminase antibodies in dermatitis herpetiformis. Gastroenterology. 1999;117(3):749-750.

34. Dieterich W, Laag E, Bruckner-Tuderman L, et al. Antibodies to tissue transglutaminase as serologic markers in patients with dermatitis herpetiformis. J Invest Dermatol. 1999;113(1):133-136.

35. Kumar V, Jarzabek-Chorzelska M, Sulej J, Rajadhyaksha M, Jablonska S. Tissue transglutaminase and endomysial antibodiesdiagnostic markers of gluten-sensitive enteropathy in dermatitis herpetiformis. Clin Immunol. 2001;98(3):378-382.

36. Desai AM, Krishann RS, Hsu S. Medical pearl: using tissue transglutaminase antibodies to diagnose dermatitis herpetiformis. $\mathrm{J} \mathrm{Am} \mathrm{Acad}$ Dermatol. 2005;53(5):867-868

37. Alonso-Llamazares J, Gibson LE, Rogers RS 3rd. Clinical, pathologic, and immunopathologic features of dermatitis herpetiformis: review of the Mayo Clinic experience. Int J Dermatol. 2007;46(9):910-919.

38. Kagnoff MF. AGA Institute medical position statement on the diagnosis and treatment of celiac disease. Gastroenterology. 2006;131(6): 1977-1980.

39. Giersiepen K, Lelgemann M, Stuhldreher N, et al. Accuracy of diagnostic antibody tests for coeliac disease in children: summary of an evidence report. J Pediatr Gastroenterol Nutr. 2012;54(2):229-241.

40. Lewis NR, Scott BB. Meta-analysis: deamidated gliadin peptide antibody and tissue transglutaminase antibody compared as screening tests for coeliac disease. Aliment Pharmacol Ther. 2010;31(1):73-81.

41. Prause C, Ritter M, Probst C, et al. Antibodies against deamidated gliadin as new and accurate biomarkers of childhood coeliac disease. J Pediatr Gastroenterol Nutr. 2009;49(1):52-58.

42. Agardh D. Antibodies against synthetic deamidated gliadin peptides and tissue transglutaminase for the identification of childhood celiac disease. Clin Gastroenterol Hepatol. 2007;5(11):1276-1281.

43. Liu E, Li M, Emery L, et al. Natural history of antibodies to deamidated gliadin peptides and transglutaminase in early childhood celiac disease. J Pediatr Gastroenterol Nutr. 2007;45(3):293-300.

44. Jaskowski TD, Donaldson MR, Hull CM, et al. Novel screening assay performance in pediatric celiac disease and adult dermatitis herpetiformis. J Pediatr Gastroenterol Nutr. 2010;51(1):19-23.

45. Sugai E, Hwang HJ, Vázquez H, et al. New serology assays can detect gluten sensitivity among enteropathy patients seronegative for antitissue transglutaminase. Clin Chem. 2010;56(4):661-665.

46. Lytton SD, Antiga E, Pfeiffer S, et al. Neo-epitope tissue transglutaminase autoantibodies as a biomarker of the gluten sensitive skin diseasedermatitis herpetiformis. Clin Chim Acta. 2013;415:346-349.

47. Sárdy M, Kárpáti S, Merkl B, Paulsson M, Smyth N. Epidermal transglutaminase (TGase 3) is the autoantigen of dermatitis herpetiformis. J Exp Med. 2002;195:747-757.

48. Hull CM, Liddle M, Hansen N, et al. Elevation of IgA anti-epidermal transglutaminase antibodies in dermatitis herpetiformis. BrJ Dermatol. 2008;159(1):120-124.

49. Jaskowski TD, Hamblin T, Wilson AR, et al. IgA anti-epidermal transglutaminase antibodies in dermatitis herpetiformis and pediatric celiac disease. J Invest Dermatol. 2009;129(11):2728-2730.

50. Borroni G, Biagi F, Ciocca O, et al. IgA anti-epidermal transglutaminase autoantibodies: a sensible and sensitive marker for diagnosis of dermatitis herpetiformis in adult patients. J Eur Acad Dermatol Venereol. 2013;27(7):836-841.

51. Kasperkiewicz M, Dähnrich C, Probst C, et al. Novel assay for detecting celiac disease-associated autoantibodies in dermatitis herpetiformis using deamidated gliadin-analogous fusion peptides. $\mathrm{J} \mathrm{Am} \mathrm{Acad}$ Dermatol. 2012;66(4):583-588.

52. Oberhuber G, Granditsch G, Vogelsang H. The histopathology of coeliac disease: time for a standardized report scheme for pathologists. Eur $J$ Gastroenterol Hepatol. 1999:11(10):1185-1194.

53. Rubio-Tapia A, Hill ID, Kelly CP, Calderwood AH, Murray JA; American College of Gastroenterology. ACG clinical guidelines: diagnosis and management of celiac disease. Am J Gastroenterol. 2013;108(5):656-676.
54. Bai JC, Fried M, Corazza GR, et al. World Gastroenterology Organisation global guidelines on celiac disease. J Clin Gastroenterol. 2013; 47(2):121-126

55. Rottmann LH. Details of the gluten-free diet for the patients with dermatitis herpetiformis. Clin Dermatol. 1992;9(3):409-414.

56. Turchin I, Barankin B. Dermatitis herpetiformis and gluten-free diet. Dermatol Online J. 2005;11(1):6.

57. Garioch JJ, Lewis HM, Sargent SA, Leonard JN, Fry L. 25' years experience of a gluten-free diet in the treatment of dermatitis herpetiformis. Br J Dermatol. 1994;131(4):541-545.

58. Lewis HM, Renaula TM, Garioch JJ, et al. Protective effect of glutenfree diet against development of lymphoma in dermatitis herpetiformis. Br J Dermatol. 1996;135(3):363-367.

59. Lemberg D, Day AS, Bohane T. Coeliac disease presenting as dermatitis herpetiformis in infancy. J Pediatr Child Health. 2005;41(5-6): 294-296.

60. Hardman CM, Garioch JJ, Leonard JN. Absence of toxicity of oats in patients with dermatitis herpetiformis. N Engl J Med. 1997;337(26): 1884-1887.

61. Janatuinen BK, Pikkarainen SA, Kemppainen TA. A comparison of diets with and without oats in adults with celiac disease. $N$ Engl J Med. 1995;333(16):1033-1037.

62. Mäki M. Celiac disease treatment: gluten-free diet and beyond. J Pediatr Gastroenterol Nutr. 2014;59(Suppl 1):S15-S17.

63. Hischenhuber C, Crevel R, Jarry B, et al. Safe amounts of gluten for patients with wheat allergy or coeliac disease. Aliment Pharmacol Ther. 2006;23(5):559-575.

64. Bardella MT, Fredella C, Trovato C, et al. Long-term remission in patients with dermatitis herpetiformis on a normal diet. Br J Dermatol. 2003;149(5):968-971.

65. Paek SY, Steinberg SM, Katz SI. Remission in dermatitis herpetiformis: a cohort study. Arch Dermatol. 2011;147(3):301-305.

66. Antiga E, Caproni M, Pierini I, Bonciani D, Fabbri P. Gluten-free diet in patients with dermatitis herpetiformis: not only a matter of skin. Arch Dermatol. 2011;147(8):988-989.

67. Fric P, Gabrovska D, Nevoral J. Celiac disease, gluten-free diet, and oats. Nutr Rev. 2011;69(2):107-115.

68. Fasano A. Novel therapeutic/integrative approaches for celiac disease and dermatitis herpetiformis. Clin Dev Immunol. 2012;2012:959061.

69. Tuire I, Marja-Leena L, Teea S, et al. Persistent duodenal intraepithelial lymphocytosis despite a long-term strict gluten-free diet in celiac disease. Am J Gastroenterol. 2012;107(10):1563-1569.

70. Sener O, Doganci L, Safali M, Besirbellioglu B, Bulucu F, Pahsa A. Severe dapsone hypersensitivity sindrome. J Invest Allergol Clin Immunol. 2006;16(4):268-270.

71. Kosann MK. Dermatitis herpetiformis. Dermatol Online J. 2001;9(4):8.

72. Talarico DF, Metro DG. Presentation of dapsone-induced methemoglobinemia in a patient status post-bowel transplant. $J$ Clin Anesth. 2005;17(7):568-570.

73. Lee I, Barton TD, Goral S, et al. Complications related to dapsone use for Pneumocystis jirovecii pneumonia prophylaxis in solid organs transplant recipients. Am J Transplant. 2005;5(11):2791-2795.

74. Damodar S, Viswabandya D, George B, Mathews V, Chandy M, Srivastava A. Dapsone for chronic idiopathic thrombocytopenic purpura in children and adults - a report of 90 patients. Eur J Hematol. 2005; 75(4):328-331.

75. Alves-Rodrigues EN, Ribeiro LC, Dioz Silva M, Takiuchi A, Fontes CJ. Dapsone syndrome with acute renal failure during leprosy treatment: case-report. Braz J Infect Dis. 2005;9(1):84-86.

76. Chalioulias K, Mayer E, Darvay A, Antcliff R. Anterior ischaemic optic neuropathy associated with dapsone. Eye. 2006;20(8):943-945.

77. Mery L, Dega H, Prost C, Dubertret L. Polynévrite sensitive induite par la dapsone (Disulone ${ }^{\circledR}$ ) [Dapsone-induced sensory peripheral neuropathy]. Ann Dermatol Venereol. 2003;130(4):447-449. French.

78. Bolotin D, Petronic-Rosic V. Dermatitis herpetiformis. Part II. Diagnosis, management, and prognosis. J Am Acad Dermatol. 2011; 64(6):1027-1033. 
79. McFadden JP, Leonard JN, Powles AV, Rutman AJ, Fry L. Sulphamethoxypyridazine for dermatitis herpetiformis, linear IgA disease and cicatricial pemphigoid. Br J Dermatol. 1989;121(6):759-762.

80. Willsteed E, Lee M, Wong LC, Cooper A. Sulfasalazine and dermatitis herpetiformis. Australas J Dermatol. 2005;46(2):101-103.

81. Shah SA, Ormerod AD. Dermatitis herpetiformis effectively treated with heparin, tetracycline and nicotinamide. Clin Exp Dermatol. 2000;25(3):204-205.

82. Alexander JO. The treatment of dermatitis herpetiformis with heparin. Br J Dermatol. 1963;75:289-293.

83. Stenveld HJ, Starink TM, van Joost T, StoofTJ. Efficacy of cyclosporine in two patients with dermatitis herpetiformis resistant to conventional therapy. J Am Acad Dermatol. 1993;28(6):1014-1015.

84. Johnson HH Jr, Binkley GW. Nicotinic acid therapy of dermatitis herpetiformis. J Invest Dermatol. 1950;14(4):233-238.

85. Zemtsov A, Neldner KH. Successful treatment of dermatitis herpetiformis with tetracycline and nicotinamide in a patient unable to tolerate dapsone. J Am Acad Dermatol. 1993;28(3):505-506.
86. Silvers DN, Juhlin EA, Berczeller PH, McSorley J. Treatment of dermatitis herpetiformis with colchicine. Arch Dermatol. 1980;116(12): 1373-1384.

87. Kotze LM. Dermatitis herpetiformis, the celiac disease of the skin! Arq Gastroenterol. 2013;50(3):231-235.

88. Schmidt E. Therapieoptimierung bei schweren bullösen Autoimmundermatosen [Optimizing therapy in patients with severe autoimmune blistering skin diseases]. Hautarzt 2009;60(8):633-640. German.

89. Antiga E, Quaglino P, Pierini I, et al. Regulatory T cells as well as IL-10 are reduced in the skin of patients with dermatitis herpetiformis. J Dermatol Sci. 2015;77(1):54-62.

90. National Institutes of Health consensus development conference statement on celiac disease, June 28-30, 2004. Gastroenterology. 2005;128(4 Suppl 1):S1-S9.

\section{Publish your work in this journal}

Clinical, Cosmetic and Investigational Dermatology is an international, peer-reviewed, open access, online journal that focuses on the latest clinical and experimental research in all aspects of skin disease and cosmetic interventions. All areas of dermatology will be covered; contributions will be welcomed from all clinicians and basic science researchers globally. This journal is indexed on CAS. The manuscript management system is completely online and includes a very quick and fair peer-review system, which is all easy to use. Visit http://www.dovepress.com/testimonials.php to read real quotes from published authors.

Submit your manuscript here: http://www.dovepress.com/clinical-cosmetic-and-investigational-dermatology-journal 